\title{
Deutschland unterwegs
}

\section{Zeitverwendung für Mobilität im wiedervereinten Deutschland}

\section{Caroline Kramer, München}

\section{Individuelle Zeitverwendung als geographisches Thema}

«Rasender Stillstand» - diese Vision prognostizierte ViriLIo (1992), in der sich die Menschheit mit Hilfe der weltweit vernetzten Computer in eine paradoxe Situation des einerseits beschleunigten Lebens bei gleichzeitig zunehmender physischer Unbeweglichkeit manövriere. Er stellt die These auf, dass durch digitale Kommunikationstechnologien die reale Welt von der digitalen Welt abgelöst werde und damit auch die reale Mobilität zunehmend von der virtuellen Mobilität verdrängt werde. GUGGENBERGER (1997) sagt sogar voraus, dass «die geographische Ordnung... von der chronographischen Ordnung verdrängt (werde)» (1997: 22). Im Hinblick auf diese Prognosen stellt sich die Frage: Sind Anzeichen dieser Zukunftsvisionen bereits erkennbar? Nimmt die reale Mobilität tatsächlich ab? Wird die Bedeutung des Raums von der Übermacht der Zeit bereits abgelöst?

Der Diskurs zum Umgang mit Zeit im/und Raum ist ein Thema, das mittlerweile sämtliche räumlichen Maßstabsebenen berührt. Auf gesamtgesellschaftlicher Ebene ist die Diskussion um das Zeitverständnis und Zeitbewusstsein bereits seit geraumer Zeit lebendig (RAMMSTEDT 1975; MüLleR-WICHMANN 1984; RINDERSPACHER 1985; HeNCKel et al. 1989; LÜDTKE 1995; GarhamMER 1996), und auf der Ebene der Städte und Kommunen sind vor allem Projekte des Deutschen Instituts für Urbanistik (HencKel et al. 1997, 2001; EBERLING 1999, 2002), der Projektgruppe «zeiten:der:stadt» (MücKENBERGER 1999, 2001) und des Arbeitskreises «Räumliche Wirkungen veränderter Zeitstrukturen» der Akademie für Raumforschung und Landesplanung (ARL) (HENCKEL \& Eberling 2002) sowie von Wolf \& Scholz (1999) zu nennen. Die Diskussion über den individuellen Umgang mit Zeit findet ihren Niederschlag in einer seit einigen Jahren stärker werdenden Flut von Ratgebern und Artikeln, die dem angeblich gestressten Menschen der Postmoderne entweder professionelle Tipps zum noch effektiveren Zeit-Management oder zur gezielten Abkehr von fremdbestimmtem Zeitdruck und der (Wieder-) Entdeckung von Langsamkeit und Muße geben.

\subsection{Zeit und Raum in der klassischen time geography}

Zeit und Raum ist ein Thema, das in der Geographie in den 1970er und 1980er Jahren in Form der time geography (HäGERSTRAND 1970, 1975, 1978; CARLSTEIN, PARKES \& THRIFT 1978) vor allem im englischsprachigen Raum, aber auch in Deutschland - z.T. im Rahmen der Aktionsraumforschung (KLINGBEIL 1978, 1980; SCHreiner 1995) - intensiv bearbeitet wurde. HaLLiN (1991) sah darüber hinaus in der Ergänzung der klassischen Arbeiten der time geography um handlungstheoretische Elemente eine Möglichkeit, die bisherigen Ansätze in ein modernes sozialgeographisches Konzept zu überführen. Die Kritik an der Zeitgeographie richtete sich neben dem Vorwurf einer zu starken Vereinfachung, der Theorielosigkeit und des Holismus vor allem dagegen, dass sie von einem rational zweckorientiert handelnden Menschen ausgehe, dessen Handeln nach einer "Zeit-Nutzen-Funktion" ablaufe (KLINGBEIL 1980:22). Ein weiterer Kritikpunkt an der traditionellen time geography ist nach ZIERHOFER (1989) die Tatsache, dass sie «weitgehend ohne begriffliche und konzeptionelle Brücke zu soziokulturellen und psychischen Prozessen geblieben (sei)» (Zierhofer 1989: 87). Er sieht in den handlungstheoretischen Ansätzen und der Strukturationstheorie die Stufe, auf der Handlungen als Elemente der sozialen Realität betrachtet werden und damit auch constraints als Ergebnis von Handlungsfolgen einzustufen seien. In der Weiterführung der Konzepte der time geography in GidDENS' Strukturationstheorie (GIDDENS 1995) sieht er - ähnlich wie CARLSTEIN (1981) und HaLLIN (1991) - durchaus Möglichkeiten für eine verbesserte Anwendbarkeit dieses Ansatzes.

Als weiteres ungelöstes Problem der klassischen time geography bzw. Aktionsraumforschung kommt hinzu, dass sich die empirischen Arbeiten bisher auf ein begrenztes Gebiet, wie z.B. bestimmte Ortsteile einer Großstadt oder den Einzugsbereich eines zentralen Ortes, beschränken mussten, so dass die Analysen auf der Mikro- oder Mesoebene verharrten und die Makroebene nicht erreichten. Hinzu kommt, dass das hoch gesteckte Ziel einiger Aktionsraumstudien, die alles erklärende Theorie menschlichen Verhaltens im Raum zu entwickeln, scheiterte. Sowohl die Ansätze der geographischen als auch die der (stadtsoziologischen) Aktionsraumforschung gelangten immer wieder an den Punkt, an dem auch bei großen Datenmengen Ursachen, Gründe und Motive für die Auswahl bestimmter Gelegenheiten und Verkehrsmittel im Dunkeln blieben.

1.2 Raum-zeitlicher Kontext in der Sozialgeographie In den Ansätzen der handlungszentrierten Sozialgeo- 
graphie stellt der raum-zeitliche Kontext nun nicht mehr die Voraussetzung menschlichen Handelns - wie in der time geography - sondern das Ergebnis menschlichen Handelns dar (WERLEN 2000). Dennoch besitzt er innerhalb der handlungszentrierten Perspektive als ein Element neben anderen eine gewisse Relevanz, obwohl seine zeitliche Komponente in dieser Diskussion deutlich in den Hintergrund getreten ist. In dem hier vorgestellten Projekt wird der Kontext als Bündel von - zum größten Teil von Menschen geschaffenen constraints und Möglichkeiten verstanden. Dabei wird davon ausgegangen, dass zum einen die Akteure ihren Kontext gestalten (z.B. durch ihre PKW-Nutzung Staus produzieren sowie langfristig neue Straßen verursachen), zum anderen aber auch dieser durch (frühere) Akteure gestaltete aktuelle Kontext Bedingung oder constraint ihres Lebensalltags darstellt. Somit ist das Verständnis von Kontext hier nicht deterministisch, sondern probabilistisch und zudem im Sinne von WEICHHART (2003) als action setting zu verstehen (aufsetzend u.a. auf BARKERS "Setting-Theorie» (1968)). Wie sich Mobilitätsmuster entlang der Eigenschaften des Kontexts und/oder denen der Akteure identifizieren lassen, stand im Mittelpunkt des Projekts zum Thema "Zeit und Mobilität», aus dem hier eine Auswahl der Ergebnisse vorgestellt wird (KRAMER 2000, 2004). Es liegt nicht in der Absicht der Autorin, auf dem begrenzten Raum einen vollständigen Überblick über den Stand der Forschung der time geography zu bieten. Ebenso wenig können alle Ergebnisse des empirischen Teils präsentiert werden. Was dieser Beitrag jedoch beabsichtigt, ist, das Augenmerk auf eine $\mathrm{zu}$ Unrecht in den Hintergrund geratene Forschungsrichtung zu lenken und Chancen neuer Auswertungsmöglichkeiten aufzuzeigen.

\section{Kontext und Akteur im Wechselspiel - Fragestellung und Methoden}

In diesem Beitrag soll nun anhand der Zeitverwendung für Mobilität ein Konzept entwickelt werden, das neue Anwendungsmöglichkeiten einer modifizierten time geography vorstellt. Ausgehend von einem relationalen Raumverständnis (nach BLotevogel 1995: 735), in dem ein Zeitmaß stellvertretend als Raummaß eingesetzt wird, soll hier die Zeit, die für Wege im Alltag verwendet wird, als Distanzmaß Anwendung finden. Mobilitätszeit variiert dabei zum einen über den Kontext (im Sinne von setting, d.h. im Wesentlichen von gestaltetem, konstruiertem Kontext, in dem sich die Akteure nach bestimmten Regeln bewegen) und zum anderen über die Akteure, die in einem komplexen Wechselspiel miteinander stehen. Hinzu kommt, dass die Gestaltung der alltäglichen (kurzfristigen) Mobilität in hohem Maße von früheren bereits gefällten (langfristigen) Mobilitätsentscheidungen abhängig ist.
Die zentrale Frage lautet somit: Ist Zeit als Ressource nicht nur sozial, sondern auch regional ungleich verteilt? Genauer formuliert: Gibt es Wechselwirkungen zwischen den (von Akteuren konstruierten) Eigenschaften des Kontexts (räumlich, gesellschaftlich) auf der Makroebene und der individuellen Zeitverwendung von Mobilität der Akteure auf der Mikroebene und wie sehen sie aus?

Die zentralen Forschungsfragen, die in diesem Beitrag zu Mobilität im wiedervereinten Deutschland behandelt werden sollen, lauten demzufolge:

- Wie gestalten sich die Wegezeiten im Alltag für die Akteure in ihrem Kontext? Also, wer hat im Alltag wo für welche Zwecke die längeren und wer die kürzeren Wege?

- Welchem Wandel unterliegen die Wegezeiten, vor allem seit der Wiedervereinigung in den neuen und alten Ländern? Dieser Vergleich zwischen den alten und neuen Ländern soll die Frage beantworten, ob die unterschiedlichen politischen Systeme in den alten und neuen Ländern als gesellschaftlicher Kontext heute noch auf einzelne Elemente des Gesamtsystems Mobilität nachwirken.

\subsection{Daten zur Zeitverwendung und zum Kontext}

Diesen Fragen wird im Folgenden mit Hilfe der beiden bundesdeutschen Zeitbudgetstudien nachgegangen. Mit der ersten bundesdeutschen Zeitbudgetstudie 1991/92 und ihrer Nachfolgestudie 2001/02 ist seit relativ kurzer Zeit eine bisher einmalige Datenbasis zugänglich, die es erlaubt, erstmals bundesweite Analysen der individuellen Zeitverwendung und ihrer Entwicklung seit der deutschen Wiedervereinigung durchzuführen.

Die Methoden, mit denen man die Zeitverwendung von Menschen in ihrem Alltag untersuchen kann, sind vielfältig. Besonderen Stellenwert nimmt dabei das Zeitbudgetprotokoll ein, auch Tagebuchmethode genannt (nach BLass 1980:86f.). Es gilt als «Königsweg» der Zeiterhebungsmethoden, da hier mit geringem zeitlichem Verzug unmittelbar die Aktivitäten in ein Tagebuch eingetragen werden können, so dass man erhofft, weniger Details zu verlieren als dies bei anderen Methoden der Fall ist. Für die Standardisierung des Protokolls wurden die zeitlichen Einheiten vorgegeben, die Aktivitäten wurden von den Befragten selbst formuliert und anschließend nach einem einheitlichen Muster codiert.

In Studien, die sich explizit der Untersuchung des Verkehrsverhaltens und der Mobilitätsmuster widmen, werden zwar präzisere Angaben zu einzelnen Aspekten der Mobilität (wie z.B. Entfernungen und Zielorte) erhoben als in allgemeinen Zeitbudgetstudien (z.B. in der «Kontinuierlichen Erhebung zum Verkehrs- 
verhalten» (KONTIV) $(1976,1982,1989,2002)$ oder dem «Mobilitätspanel» (MOP) (2002), beide im Auftrag des BUNDESMINISTERIUMS FÜR VERKEHR, BAU UND WOHNUNGSWESEN). Viele dieser Erhebungen werden jedoch entweder nicht bundesweit durchgeführt oder ihre Daten sind nicht auf der Ebene der Wohngemeinde auswertbar. Hinzu kommt, dass sie meist nicht über zusätzliche Informationen zum Haushalt (Ausstattung, Merkmale der Infrastruktur usw.) und zu den Einzelpersonen verfügen.

Für den Themenbereich Mobilität sind folgende Indikatoren aus der Zeitbudgetstudie nutzbar:Wegezweck, Wegedauer, Wegezahl, zeitlicher Beginn und Ende des Weges und das verwendete Verkehrsmittel. Zum Wegeziel und der zurückgelegten Entfernung sowie zu den Gründen für die Wahl des Verkehrsmittels werden allerdings auf Personenebene keine Informationen erhoben.

Die Operationalisierung des zentralen Begriffs «Kontext» erfolgt in der vorliegenden Arbeit u.a. unter Zuhilfenahme ergänzender Daten, wie z.B. aus dem Informationssystem "Indikatoren und Karten zur Raumentwicklung» (INKAR) des BUNDESAMTES FÜR BAUWESEN UND RaumordNung (BBR) (1998). Das zentral-örtliche Gliederungs- und Verflechtungssystem der BBR-Regionstypen bietet sich dadurch, dass Wegezeiten ein Wechselspiel aus Angebot und Nachfrage darstellen, als ein sinnvolles Konzept für die Untersuchung der Wegezeiten an, da es gewissermaßen die Angebotsstruktur abbildet. Durch die Feingliederung der Gemeindetypen bietet es zudem die Möglichkeit, Zentren und ihre suburbanen Wohngemeinden zu unterscheiden, was bei der bundesweiten Betrachtung von großer Bedeutung ist. «Kontext» wird im Folgenden - wie bereits erwähnt - als Umwelt der Akteure, im Sinne von Lage und Infrastruktur der Wohngemeinde und des Wohnumfeldes verstanden. Dazu zählen z.B. die Lage des Wohnorts in West- oder Ostdeutschland (da dies 1991/92, aber auch heute noch mit zahlreichen typischen Mobilitätsrestriktionen oder -vorteilen verbunden war/ist) oder die Zugehörigkeit zu einem Kreisoder Gemeindetyp (nach Typisierungen des BBR). Die Merkmale der Akteure sind Merkmale des Individuums, wie z.B. Alter, Geschlecht, Ausbildungsniveau oder Haushaltsform.

\subsection{Indikatoren der Zeitverwendung für Mobilität}

Die hier vorgestellten Auswertungen basieren auf verschiedenen Indikatoren. Für die Analyse der Anteile der Wegearten an allen Wegen wurde ausgezählt, in welchen Zeiteinheiten Wege für einen bestimmten Zweck durchgeführt wurden (2001/02 im 10-MinutenTakt erhoben). Die mittleren Wegezeiten selbst wurden ausschließlich für sogenannte Ausübende berechnet, d.h. Tage, an denen die Einzelpersonen keine Wege zurücklegten, wurden nicht in die Mittelwertbildung einbezogen, worin sich die vorliegenden Berechnungen von denen des Statistischen Bundesamtes unterscheiden. Unter dem Anteil der "Ausübenden" als weiterem Indikator ist der Anteil der Personen zu verstehen, die an mindestens einem der Befragungstage einen Weg für einen bestimmten Zweck zurücklegten. Für die Gegenüberstellung der Kontext- und der Akteursmerkmale wurde die Methode der Kontraste gewählte, eine lineare parametrischen Funktion, in der die Mittelwertsdifferenzen der Wegezeiten für die dichotomisierten Kontext- und Akteursmerkmale verglichen wurden.

Wie nicht selten bei empirischen Arbeiten besteht allerdings auch bei dem vorgestellten Konzept eine Kluft zwischen den «idealen» Daten und den Daten, die zur Auswertung zur Verfügung stehen. Es wäre z.B. wünschenswert, die Gemeindekennziffer sowohl für die Auswertung als auch für die Veröffentlichung nutzen zu dürfen. Ebenso wäre es für Analysen der alltäglichen Aktionsräume wichtig, Information über den Wohnstandort unterhalb der politischen Gemeinde, z.B. auf Stadtteilebene, zu besitzen. Auch die Zielgemeinde der einzelnen Wege wäre eine sinnvolle und wichtige Information. Ebenfalls wäre es ein großer Gewinn, wenn man vertiefende Interviews hinsichtlich der Qualität dieser Zeitverwendung mit den Befragten durchführen könnte. Derartige Interviews wurden zwar in dem Projekt, aus dem hier Auszüge präsentiert werden (KRAMER 2004), durchgeführt, es wäre jedoch wünschenswert gewesen, ausführlichere Interviews mit einer Auswahl der Zeitbudget-Befragten durchführen zu können. Trotz dieser Kluft zwischen Wunsch und Wirk lichkeit bietet der Datensatz der Zeitbudgetstudien zum ersten Mal die Möglichkeit, bundesweite Analysen der Zeitverwendung durchzuführen. Eine stärkere Nutzung durch Geographen und Geographinnen könnte auch dazu beitragen, Argumente für verbesserte Auswertungsmöglichkeiten oder spezifische Fragestellungen zu stützen und damit bestehende Schwachpunkte der Zeitbudgetstudien in Zukunft abzubauen.

\section{Wer ist in Deutschland wie lange unterwegs?}

Einen Überblick über die Verteilung der Wegarten an Werk- und Wochenendtagen bietet Abb. 1. An Werktagen werden am häufigsten Wege für den Haushalt und Wege für die Arbeit zurückgelegt, gefolgt von Wegen für Freizeit, Kontakte und Bildung. An Wochenendtagen gehen erwartungsgemäß die Anteile der Wege für Arbeit und Bildung zurück, dafür steigen diejenigen für Freizeit und Kontakte an, während die Wege für den Haushalt immer noch bei einem Viertel blieben, 


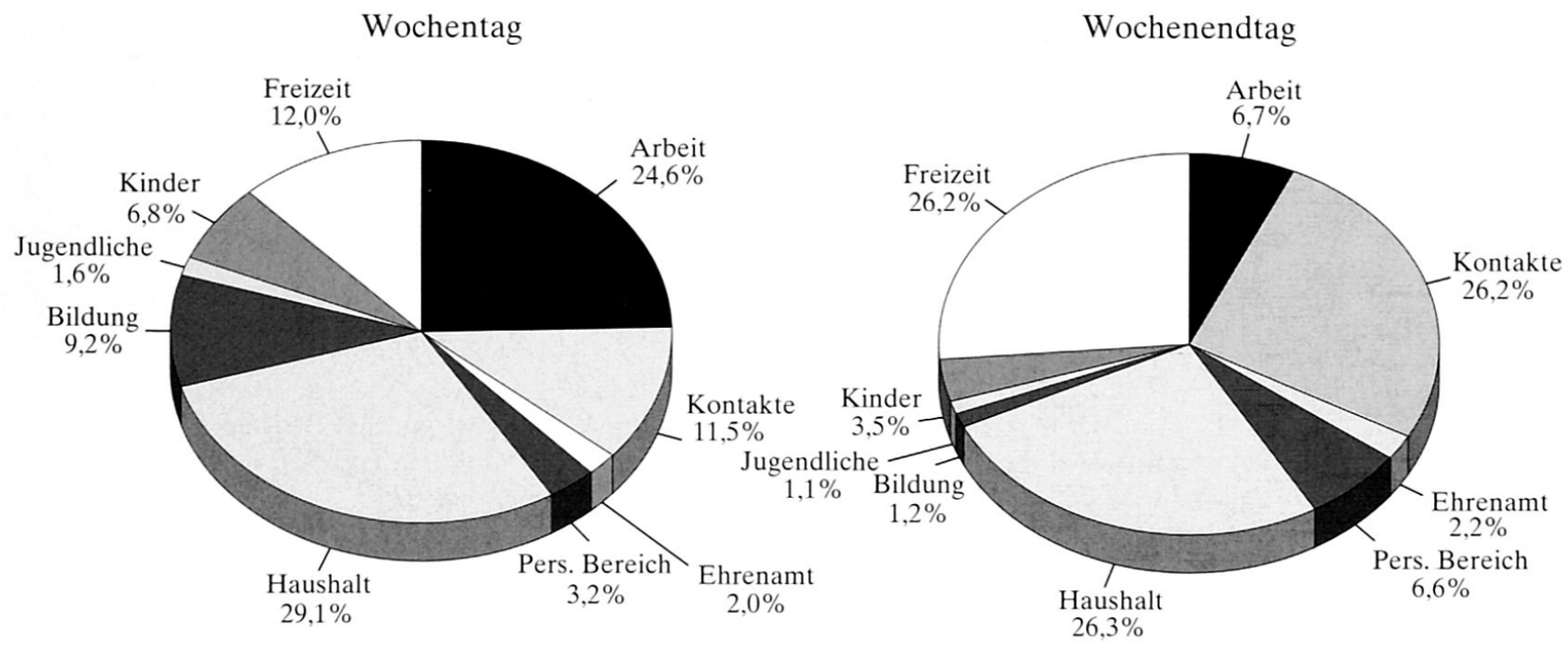

Abb. 1: Verteilung der Wegarten auf Wochentage und Wochenendtage in Deutschland 2001/02

Typological distribution of trips during the week and at the weekend in Germany 2001/02

Répartition des types de déplacements selon les jours ouvrables et les week-ends en Allemagne (2001/2002)

Quelle: Zeitbudgetstudie des Statistischen Bundesamtes 2001/02

was mit den zahlreichen Einkaufsaktivitäten an Samstagen zusammenhängt.

Für eine Beurteilung der Relevanz dieser Wegearten für das Verkehrsaufkommen als Ganzes ist es notwendig zu wissen, welche Wege besonders lang sind und welche Wege von einer Vielzahl von Personen zurückgelegt werden. In Abb. 2 wurden sowohl die Wegedauer in Minuten als auch der Anteil der Personen in Prozent, die einen jeweiligen Weg an den Befragungstagen zurückgelegt haben, dargestellt. Um der Frage nachzugehen, wie sich die Mobilität in den beobachteten zehn Jahren zwischen den beiden Erhebungen entwickelt hat, wurden die Werte beider Zeitbudgetstudien dargestellt und miteinander verbunden.

Insgesamt wird deutlich, dass die Wege für Haushalt, Arbeit, Freizeit und Kontakte zu beiden Zeitpunkten am meisten zum Verkehrsaufkommen beitrugen, da sie sowohl von einem hohen Anteil von Personen zurückgelegt wurden als auch relativ viel Zeit beanspruchten. Während die Wege für Haushaltsbesorgungen vor allem dadurch auffallen, dass sie von einem sehr hohen Anteil von Personen ausgeübt wurden, dauerten Wege für Bildung, Arbeit, Freizeit und Kontakte besonders lange; allerdings wurden diese nur von einem sehr geringen Anteil an Personen im Alltag zurückgelegt (in den beiden Zeitbudgetstudien wurden Kinder erst im Alter vor mindestens 12 Jahren (1991/92) bzw. 10 Jahren (2001/02) befragt). Mit großem Abstand folgten zu den o.g. vier Wegear- ten die Wege für den persönlichen Bereich, Wege für die Betreuung von Kindern und Jugendlichen oder für das Ehrenamt, die nur von relativ wenigen Personen ausgeübt wurden.

Im Vergleich der Erhebungszeitpunkte fällt auf, dass für einige Wegearten - ganz besonders Wege für den Haushalt - die Anteile derer, die an den Befragungstagen Wege zurücklegten, deutlich gestiegen sind. Dies ist zumindest teilweise auf erhebungstechnische Veränderungen zurückzuführen, da die Befragten des Jahres 2001/02 nicht nur zwei - wie 1991/92 -, sondern drei Tage lang ein Tagebuch führten, so dass sich damit die Wahrscheinlichkeit erhöhte, dass an diesen Tagen eine bestimmte Wegeart ausgeübt wurde. Davon unabhängig ist jedoch der für alle Wege geltende Anstieg der Wegezeit von mindestens 10 bis 15 Minuten. Dies stellt eine deutliche Steigerung um rund $20-30 \%$ dar, die vor dem Hintergrund früherer Untersuchungen geradezu als dramatisch zu bezeichnen ist. Bisher wurde in der Literatur von einem law of constant travelling time ausgegangen (z.B. JANELLE 1995; SChallaböck 1991, 1998; DeITERS, GräF \& LÖFFler 2001; Lötscher, Mayer \& Monheim 2001, Schmitz 2001), das besagt, dass die Menschen unabhängig von steigender Motorisierung oder höheren Geschwindigkeiten ungefähr rund eine Stunde pro Tag unterwegs seien. Dagegen stiegen die mittleren Wegezeiten in den Zeitbudgetstudien seit Beginn der 1990 Jahre von rd. 66 Minuten auf rd. 88 Minuten pro Tag an, so dass - zumindest im wiedervereinten Deutschland - diese 


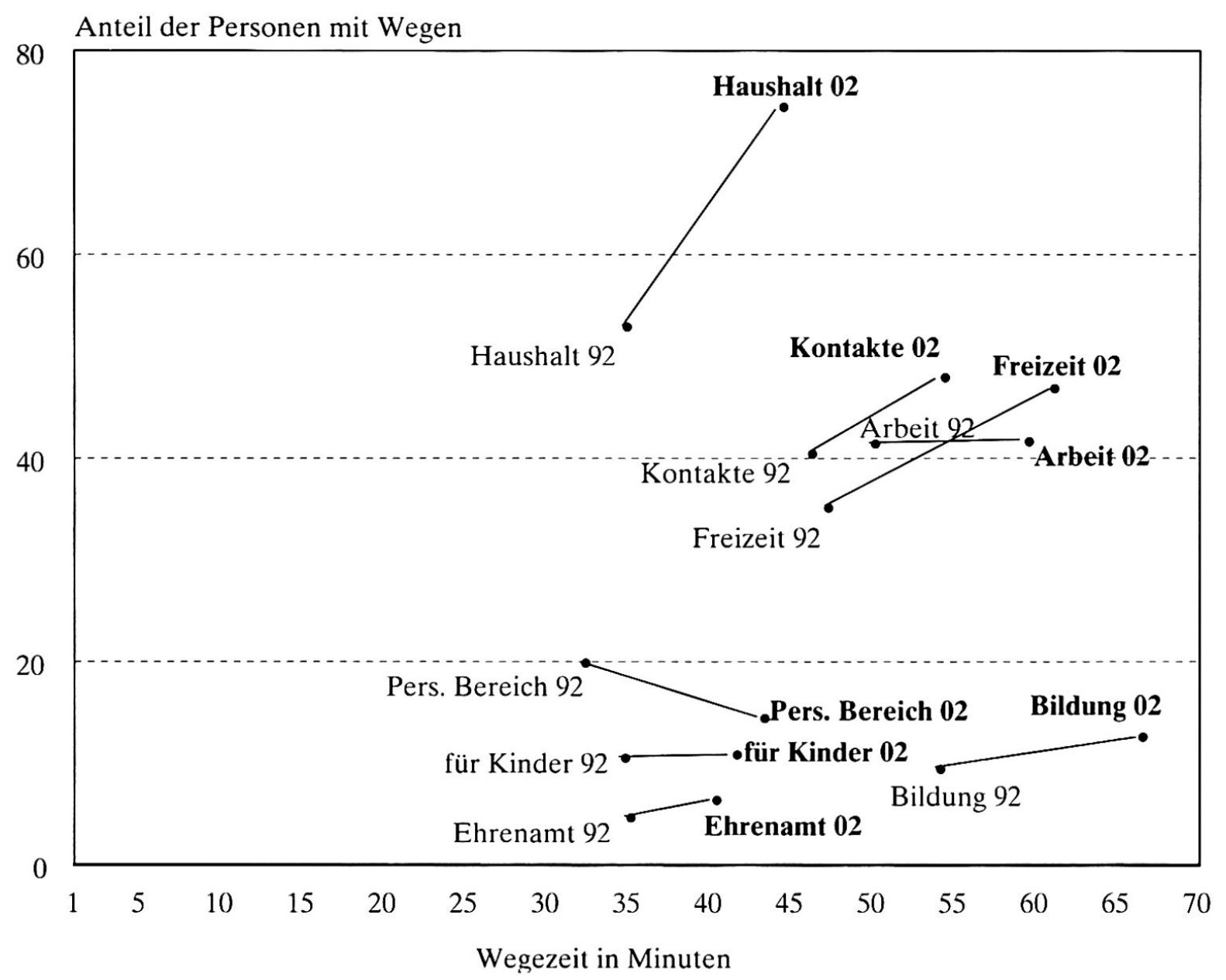

Abb. 2: Anteil derjenigen, die Wege ausführten, und die durchschnittliche Wegedauer nach Wegezweck für die Erhebungsjahre 1991/92 und 2001/02

Percentage of persons undertaking trips, as well as average trip duration according to trip purpose for the survey years 1991/92 and 2001/02

Part de ceux qui se sont déplacés et durée moyenne du trajet selon le motif du déplacement, pour les années d'investigation 1991/92 et 2001/02

Quelle: Zeitbudgetstudien des Statistischen Bundesamtes 1991/92 und 2001/02, eigene Berechnungen

bisher angenommene Regel der Konstanz der absoluten Wegezeit nicht mehr zu gelten scheint.

Besonders starke Erhöhungen erfuhren einige Wegezeiten in den neuen Bundesländern, was sich u.a. durch den Prozess der Suburbanisierung erklären lässt, durch den zunehmend Wohnstandorte außerhalb der ehemals verdichteten Städte entstanden (vgl. auch Schmitz 2001). Die hier erkennbaren Trends liefern keinerlei Hinweise auf eine Form der time-spacecompression, in der tatsächliche Mobilität letztendlich durch virtuelle Mobilität ersetzt würde, wie dies z.B. von ViriLio (1992) in seiner Vision vom «Rasenden Stillstand» vorausgesagt wurde.

Einen Vergleich der Wegezeiten und des Beteiligungs- grades zwischen West- und Ostdeutschland in ihrer zeitlichen Entwicklung bietet die Abb. 3. Wege für den Haushalt - die häufigste Wegeart - waren sowohl 1991/92 als auch 2001/02 im Osten nicht nur länger, sondern sie wurden dort auch von mehr Personen ausgeübt als im Westen. Hier spiegelt sich die höhere Beteiligung der Männer an Haushaltsaktivitäten in den neuen Bundesländern wider. Arbeitswege legte 1991/92 im Osten noch ein deutlich größerer Anteil Befragter zurück als im Westen, was mit der höheren Erwerbsbeteiligung der Frauen und dem damals insgesamt höheren Beschäftigungsgrad zusammenhängt. Die Dauer der Arbeitswege war zu diesem Zeitpunkt in Ost und West noch nahezu identisch. Diese Situation hatte sich 2001/02 grundlegend geändert: zu diesem Zeitpunkt legte ein gleich hoher Anteil der Befragten 
1991/92

$2001 / 02$

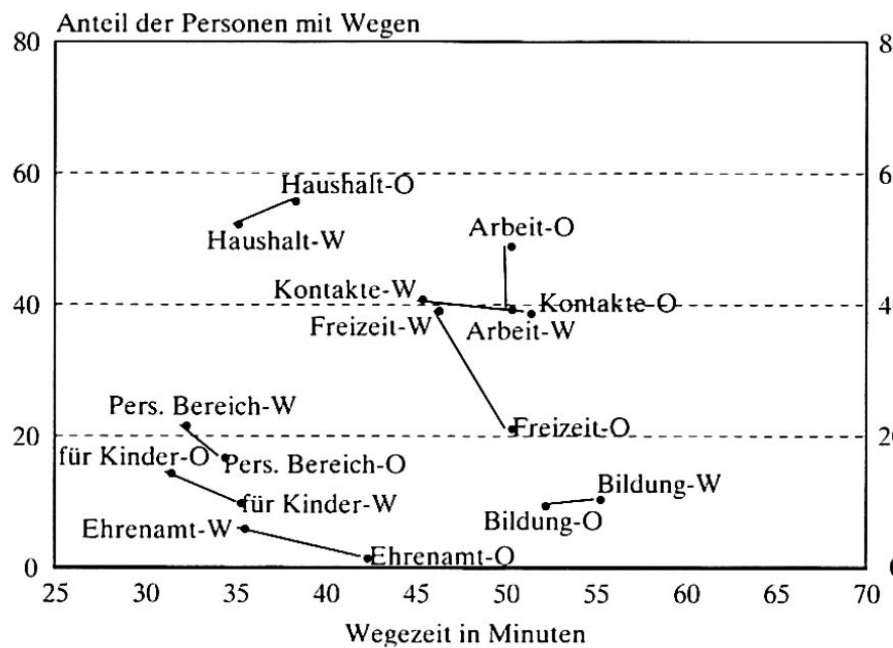

Anteil der Personen mit Wegen

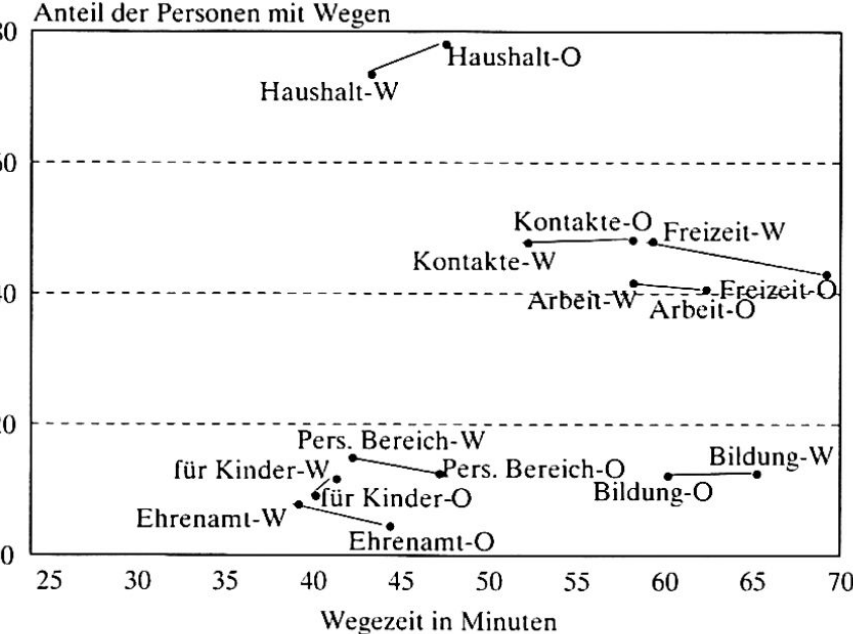

W (West), O (Ost)

Abb. 3: Anteil derjenigen, die Wege ausführten, und die durchschnittliche Wegedauer nach Wegezweck und unterschieden nach West- und Ostdeutschland 1991/92 und 2001/02

Percentage of persons undertaking trips, as well as average trip duration according to trip purpose for the survey years 1991/92 and 2001/02. Differentiation between West and East Germany.

Part des migrants et durée moyenne du trajet - Evolution comparée entre l'Allemagne de l'Ouest et l'Allemagne de l'Est (1991/92 et 2001/02)

Quelle: Zeitbudgetstudien des Statistischen Bundesamtes 1991/92 und 2001/02, eigene Berechnungen

in West- und Ostdeutschland im Alltag Wege zur Arbeit zurück, was mit der erhöhten Arbeitslosigkeit im Osten und der angestiegenen Frauenerwerbstätigkeit im Westen zusammenhängt. 2001/02 dauerten jedoch die Wege der ostdeutschen Befragten länger als die der Westdeutschen. Dies ist vermutlich darauf zurückzuführen, dass diejenigen, die bei der schwierigen Arbeitsmarktlage in den neuen Ländern noch Arbeit hatten, dafür auch relativ weite Wege in Kauf nehmen mussten (rd. ein Viertel verwendete dafür täglich mehr als 1,5 Stunden, wobei hinsichtlich des Motorisierungsgrades 2001/02 - insbesondere für Vollzeit-Erwerbstätige - keine Unterschiede zwischen West- und Ostdeutschland zu erkennen waren). Auch die Wege für Freizeitzwecke veränderten sich hinsichtlich des Beteiligungsgrades und der Wegedauer in den zehn Jahren nach der bundesdeutschen Vereinigung deutlich. Während 1991/92 nur halb so viele Ostdeutsche wie Westdeutsche im Alltag Wege für die Freizeit zurücklegten, so war dieser Unterschied 2001/02 verschwunden - in beiden Regionen nahm der Anteil derer mit Freizeitwegen zu. In den neuen Ländern erhöhten sich zudem die mittleren Wegezeiten für Freizeit so stark, so dass sie dort zu den absolut längsten Wegen zählten, d.h. Freizeitbeschäftigungen sind dort sehr wegeintensiv geworden. Die Wege für Bildung waren zu beiden Zeitpunkten unverändert im Westen (besonders in den ländlichen Regionen) länger als im
Osten. Dagegen erhöhten sich in den neuen Ländern in den beobachteten zehn Jahren die Wege für Kinderbetreuung, was z.T. auf die dortige Reduzierung der Kinderbetreuungseinrichtungen zurückzuführen ist.

Um eine Unterscheidung zwischen städtischen, verdichteten Regionen und weniger verdichteten, ländlichen Regionen zu ermöglichen, wurden mit Hilfe der 17 Gemeindetypen des Bundesamtes Für BaUwesen und Raumordnung (BBR) zwei dichotome Kategorien gebildet und diese im Zeitvergleich dargestellt (Abb. 4). Die Befragten benötigten für fast alle Wege mehr Zeit in den Städten bzw. den Verdichtungsregionen (bis auf die Wege für Bildung und Freizeit). Wege für Arbeit erforderten in den Städten sogar deutlich mehr Zeit, was u.a. darauf zurückzuführen ist, dass dort häufiger Busse und Bahnen für den Arbeitsweg genutzt wurden (vgl. auch LÖTSCHER, MAYER \& Monheim 2001) und diese in der Regel mehr Zeit beanspruchen als $\mathrm{Zu}$-Fuß-Wege oder Autofahrten. Die längeren Schulwege in den ländlichen Regionen (vor allem im Westen) blieben über die Zeit stabil, wobei in den neuen Ländern zu erwarten ist, dass die dort stattfindenden Schulschließungen im Sekundarschulbereich sich auch in längeren Schulwegen niederschlagen werden.

Besonders auffällig waren 1991/92 die in den Ver- 
$1991 / 92$

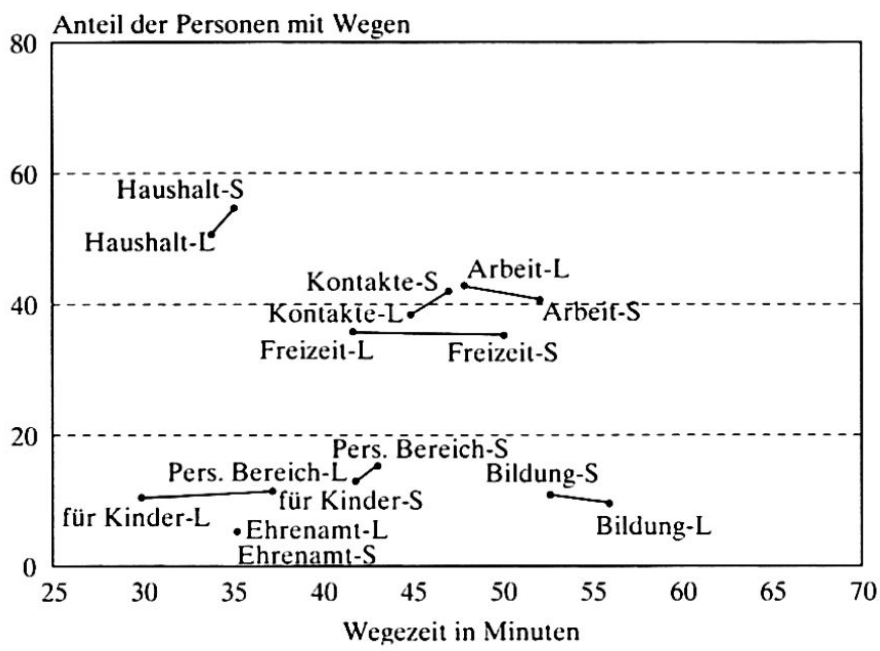

2001/02

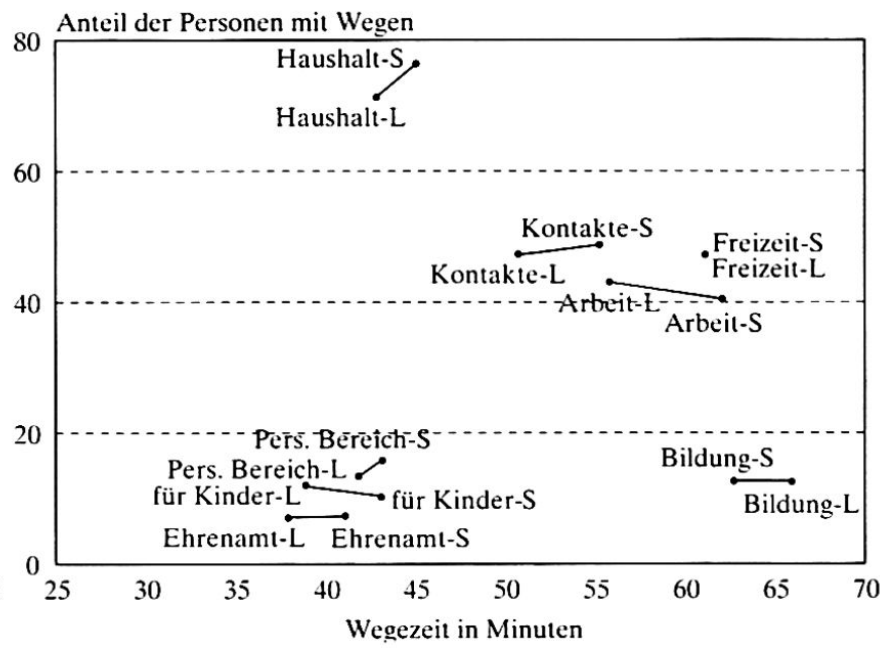

S (Stadt): Verdichtungsregion und Kernstädte der Regionen mit Verdichtungsansätzen, L (Land): restliche Gemeinden der Regionen mit Verdichtungsansätzen und ländliche Regionen

Abb. 4: Anteil derjenigen, die Wege ausführten, und die durchschnittliche Wegedauer nach Wegezweck und unterschieden nach Stadt-Land 1991/92 und 2001/02

Percentage of persons undertaking trips, as well as average trip duration according to trip purpose for the survey years 1991/92 and 2001/02. Differentiation between urban and rural regions.

Part des migrants et durée moyenne du trajet, selon le motif du déplacement - Evolution comparée entre aires urbaines et aires rurales (1991/92 et 2001/02)

Quelle: Zeitbudgetstudien des Statistischen Bundesamtes 1991/92 und 2001/02, eigene Berechnungen

dichtungsregionen (vor allem im Osten) längeren Wege für Freizeitaktivitäten. Diese Differenz hatte sich 2001/02 vollständig aufgehoben, was in hohem Maße auf die stärkere Motorisierung der Haushalte mit hohen Freizeitaktivitäten in den neuen Ländern (Haushalte mit jüngeren und erwerbstätigen Personen) und die im Vergleich zur ÖPNV-Nutzung kürzeren PKW-Wege zurückzuführen ist. $\mathrm{Zu}$ beiden Zeitpunkten dauerten die Arbeitswege und auch die Wege für Kinderbetreuung ebenfalls in den eher städtisch geprägten Verdichtungsregionen länger. Dies hing mit mehreren Faktoren zusammen: Es wurden Wege zur Arbeit in den Verdichtungsregionen von deutlich mehr Befragten (Frauen, vor allem im Osten) mit dem ÖNPV zurückgelegt und diese beanspruchten relativ viel Zeit. Die Nutzung des ÖPNVs ging in Studien aus den USA oder Großbritannien ebenfalls mit deutlich erhöhten Fahrtzeiten einher (SHEN 2000; TURNER \& GRIECO 2000).

Außerdem wurden in den zentralen Orten der ländlichen Regionen relativ viele Arbeitswege zu Fuß bzw. mit einer kurzen PKW-Fahrt zurückgelegt, so dass hier zeitlich kurze Arbeitswege entstanden. Die längeren Schulwege in den ländlichen Regionen (vor allem im Westen) sind ebenfalls in beiden Darstellungen deutlich zu erkennen. Die hier vorgestellten Ergeb- nisse decken sich mit Beobachtungen aus Arbeiten von JaNElle (1995), der die stärksten Auswirkungen der «Zeit-Raum-Konvergenz» - ausgelöst durch die flächendeckende Motorisierung - in der Peripherie und nicht in den Städten selbst feststellte (JANELLE 1995: 412).

Um der Frage nachzugehen, inwieweit Unterschiede der Wegezeiten und des Beteiligungsgrades in Abhängigkeit von Akteursmerkmalen variieren, soll an dieser Stelle exemplarisch die Variation der Wegezeiten über das Merkmal Geschlecht näher betrachtet werden (Abb. 5). Dabei werden die durch geschlechtsspezifische Rollen unterschiedlich verteilten Aufgaben im Alltag sowohl in der Wegelänge als auch - ganz besonders - in dem Anteil der ausübenden Personen deutlich. Die größten Unterschiede im Beteiligungsgrad bestanden zu beiden Zeitpunkten für Arbeitswege (die von Männern deutlich häufiger zurückgelegt wurden) und für Haushaltswege (die von Frauen deutlich häufiger zurückgelegt wurden). Auch die Wege für Kinder stellten erwartungsgemäß eher «Frauensache» dar, wobei diese Rollenunterschiede in den Wegeaktivitäten im Westen wesentlich stärker zutage traten als im Osten. Im Zeitvergleich nahm der Abstand im Beteiligungsgrad zwischen den Geschlechtern ab, was z.T. der erhöhten Zahl der Erhebungs- 
$1991 / 92$

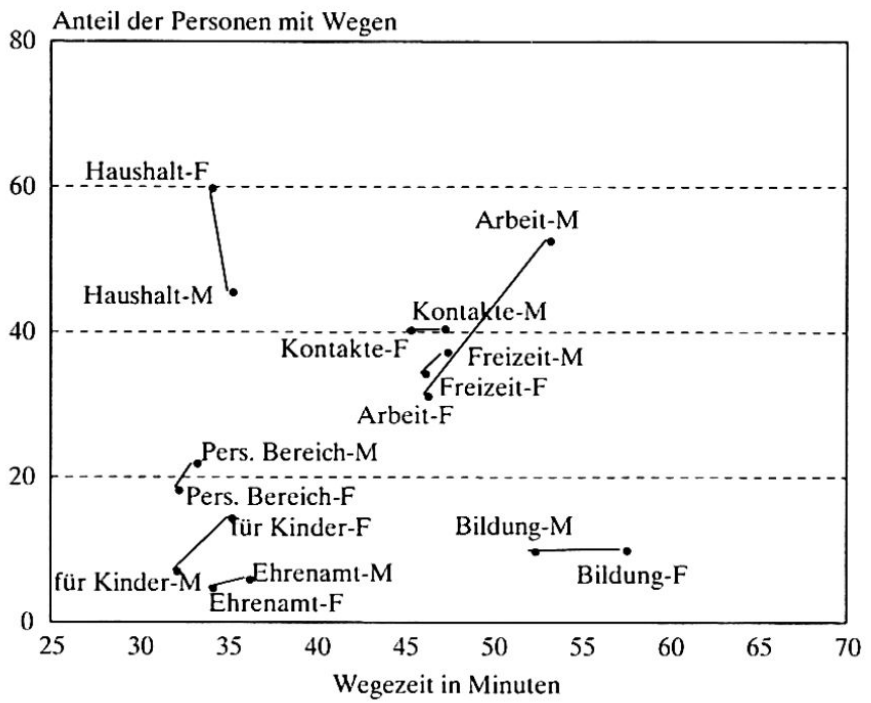

$2001 / 02$

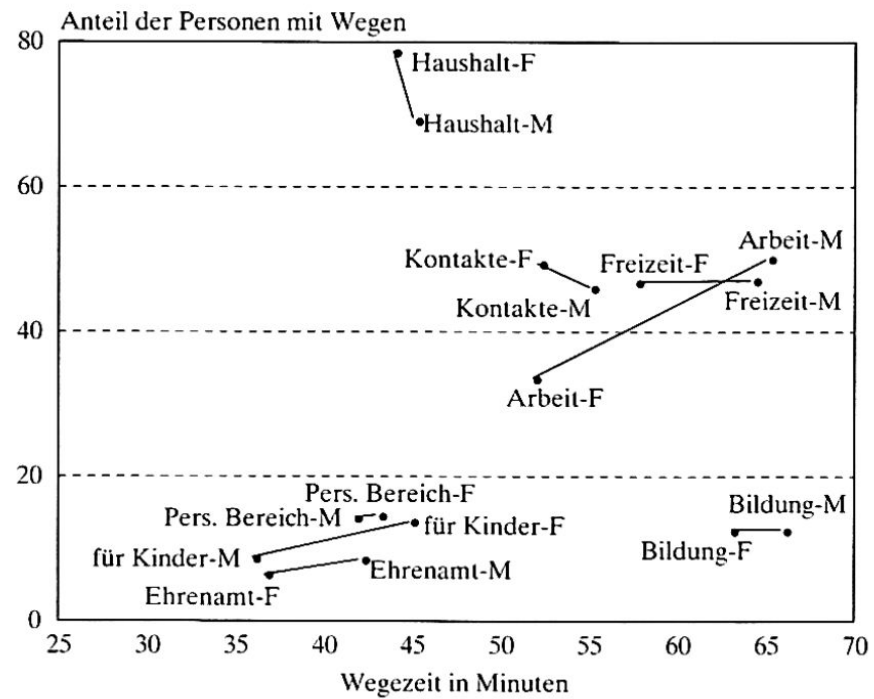

M (Männer), F (Frauen)

Abb. 5: Anteil derjenigen, die Wege ausführten, und die durchschnittliche Wegedauer nach Wegezweck und unterschieden nach Geschlecht 1991/92 und 2001/02

Percentage of persons undertaking trips, as well as average trip duration according to trip purpose for the survey years 1991/92 and 2001/02. Differentiation between sexes.

Part des migrants et durée moyenne du trajet, selon le motif du déplacement et le sexe des migrants (1991/92 et 2001/02)

Quelle: Zeitbudgetstudien des Statistischen Bundesamtes 1991/92 und 2001/02, eigene Berechnungen

tage zuzuschreiben war. Durch die Erhöhung auf drei Befragungstage 2001/02 besaßen seltener ausgeübte Aktivitäten, wie z.B. von Männern zurückgelegte Wege für den Haushalt, eine höhere Wahrscheinlichkeit, in einem der Tagebuchtage zu erscheinen.

Bemerkenswert ist auch, dass dann wenn Männer Haushaltswege zurücklegten, diese etwas länger waren als die der Frauen, was u.a. auf die gemeinsamen Großeinkäufe mit dem PKW zurückzuführen war. Deutliche Unterschiede zwischen den Geschlechtern waren ebenfalls beim Zeitaufwand für Arbeitswege festzustellen: Die Wege der (vollzeit-) erwerbstätigen Männer waren deutlich länger als die der (z.T. teilzeit-) erwerbstätigen Frauen. Dieser Unterschied verstärkte sich zwischen den beiden Erhebungszeitpunkten weiter, was vor allem auf die angestiegenen Wegezeiten der erwerbstätigen Männer im Osten zurückzuführen war (über ein Viertel der vollzeiterwerbstätigen Männer wandte 2001/02 mehr als 1,5 Stunden pro Tag für den Arbeitsweg auf).

Eine feinere Differenzierung nach Akteursgruppen (Singles, Frauen/Männer mit Kindern und mit unterschiedlichem Umfang an Erwerbstätigkeit, Rentner und Rentnerinnen), die in dem o.g. Projekt vorgenommen wurde, zeigte, dass sich vor allem der
Umfang der Erwerbstätigkeit und das Vorhandensein von Kindern im Haushalt in unterschiedlichen Wegezeitmustern niederschlugen. Dies lag in erster Linie darin begründet, dass im Verlauf der unterschiedlichen Lebensphasen auch unterschiedliche Wegearten anfielen. Die regionalen Verteilungsmuster der Regionen der (zeitlich) langen Wege (Verdichtungsregionen) und Regionen der (zeitlich) kurzen Wege blieben jedoch im Wesentlichen erhalten.

Die zu Beginn des Kapitels aufgeworfene Frage, ob in der bundesdeutschen Gesellschaft bereits ein Trend in Richtung «rasender Stillstand», d.h. einer Reduktion der realen Mobilität zugunsten virtueller Mobilität zu erkennen ist, lässt sich eindeutig verneinen, denn es ergaben sich für alle Wegearten Zunahmen der Wegezeiten, was im Gegensatz zu dem in der Literatur häufig postulierten law of constant travelling time steht. Inwieweit die zugehörigen Wegstrecken gleichfalls eine Verlängerung erfuhren, lässt sich anhand dieses Datenmaterials nicht eindeutig belegen. Aus anderen Arbeiten, wie z.B. MotzKus (2001), LÖTSCHER, Mayer \& Monheim (2001) oder Schmitz (2001) ist jedoch bekannt, dass sich die metrischen Wegelängen in den vergangenen Jahren kontinuierlich erhöht haben. Diese Entwicklung ging mit einer zunehmenden PKWNutzung einher, wobei besonders in den ländlichen 


\begin{tabular}{|c|c|c|c|c|}
\hline \multirow[b]{2}{*}{ Wegezeit für } & 1991/92 & 2001/02 & 1991/92 & 2001/02 \\
\hline & $\begin{array}{c}\text { Mittel } \\
\text { insgesamt } \\
\text { Wegezeit in } \\
\text { Minuten }\end{array}$ & $\begin{array}{c}\text { Mittel } \\
\text { insgesamt } \\
\text { Wegezeit in } \\
\text { Minuten }\end{array}$ & $\begin{array}{l}\text { Mittlere Differenz der } \\
\% \text {-Punkte zwischen } \\
\text { den Eigenschaften des } \\
\text { Kontexts und den } \\
\text { Eigenschaften der } \\
\text { Akteure }\end{array}$ & $\begin{array}{l}\text { Mittlere Differenz der } \\
\% \text {-Punkte zwischen } \\
\text { den Eigenschaften des } \\
\text { Kontexts und den } \\
\text { Eigenschaften der } \\
\text { Akteure }\end{array}$ \\
\hline Erwerbstätigkeit & 50,2 Minuten & 59,2 Minuten & Akteure: $+7,2 \%$ & Akteure: $+7,8 \%$ \\
\hline Haushalt & 34,4 Minuten & 44,0 Minuten & Akteure: $+4,3 \%$ & Akteure: $+1,6 \%$ \\
\hline Wege für Kinder*) & 33,4 Minuten & 41,2 Minuten & Akteure: $+2,6 \%$ & Akteure: $+12,7 \%$ \\
\hline Bildung*) & 53,7 Minuten & 64,3 Minuten & Kontext: $+9,6 \%$ & Kontext: $+4,2 \%$ \\
\hline Ehrenamt & 35,2 Minuten & 39,9 Minuten & Kontext: $+7,1 \%$ & Akteure: $+4,0 \%$ \\
\hline Kontakte & 46,1 Minuten & 53,7 Minuten & Kontext: $+0,8 \%$ & Akteure: $+0,9 \%$ \\
\hline Freizeit / Medien & 46,3 Minuten & 61,0 Minuten & Kontext: $+1,9 \%$ & Akteure: $+5,9 \%$ \\
\hline
\end{tabular}

*) nur Personen mit Kindern bzw. Personen in Ausbildung

Eigenschaften des Kontexts: West-/Ostdeutschland, Zentralität, Anbindung an die Deutsche Bundesbahn, Haustyp, Anteil Erholungsfläche, Schulendichte (Auswahl je nach Wegezweck)

Eigenschaften der Akteure: Alter, Geschlecht, Ausbildungsniveau, Erwerbstätigkeit, Haushaltsform, Kinder im Haushalt, PKW-Besitz (Auswahl je nach Wegezweck)

Tab. 1: Mittlere Differenzen in Wegezeiten für Eigenschaften des Kontexts und der Akteure (Differenzen in \% zwischen den dichotom kodierten Ausprägungen der jeweiligen Variablen)

Mean deviation of trip duration according to context and actor (deviation between the dichotomous value coded characteristics of the different variables in percent)

Différences moyennes de la durée du trajet, en fonction des caractéristiques du contexte et des acteurs. Différences en \% entre les expressions dichotomiques codées des variables respectives

Quelle: Zeitbudgetstudien des Statistischen Bundesamtes 1991/92 und 2001/02, eigene Berechnungen

Regionen und in kleinen Gemeinden große Wegeanteile mit dem PKW zurückgelegt wurden, was sich in den hier analysierten Zeitbudgetdaten bestätigen ließ.

\section{Gibt es Orte der langen und Orte der kurzen Wege?}

Um der in Kap. 2 aufgeworfenen Frage nachzugehen, ob die Wegezeiten stärker danach variieren, wo die Personen leben oder wer die Personen sind, wurde die Methode der Kontraste angewandt. Mit dieser Vorgehensweise wird erkennbar, für welche Indikatoren größere Unterschiede innerhalb der Kontextmerkmale und für welche Indikatoren größere Unterschiede innerhalb der Akteursmerkmale auftreten. In Tab. 1 sind diese mittleren Differenzen der Eigenschaften des Kontexts und der Akteure für die Wegezeiten dargestellt. Dazu wurde der Prozentanteil, den die jeweilige Differenz am gesamten Mittelwert der Wegezeit ausmacht, errechnet und die Differenz zwischen der gesamten Gruppe der Kontext- und Akteursmerkmale dargestellt.

Für die Wege zur Erwerbsarbeit waren sowohl für die Wegezeit als auch für den Anteil der Ausübenden zu beiden Zeitpunkten die Merkmale auf Akteursebene von größerer Relevanz als die Merkmale des Kontexts. Besonders wirksam waren hierbei die Unterschiede zwischen den Geschlechtern (Männer waren häufiger erwerbstätig als Frauen (vor allem im Westen) und legten längere Wege zurück). Durch den Rückgang der Anteile der Personen mit Arbeitswegen (vor allem der Männer) in den neuen Ländern bei gleichzeitigem Anstieg der Anteile der erwerbstätigen Frauen im Westen gewann das Merkmal Alter an Bedeutung. Allerdings waren auch Unterschiede hinsichtlich der Wegezeit zwischen Ost und West und zwischen Ver- 
dichtungsregionen und ländlichen Regionen zu erkennen, die jedoch im Vergleich zu den Akteursmerkmalen geringer ausgeprägt waren. Diese Unterschiede blieben über die Jahre hinweg unverändert. Die Wege für den Haushalt und Kinderbetreuung wurden erwartungsgemäß stärker durch Akteurs- als durch Kontextmerkmale bestimmt, wobei bei den Haushaltswegen der Einfluss der Akteursmerkmale abnahm (zunehmende Beteiligung der Männer), während bei den Wegen für die Kinderbetreuung dieser Einfluss deutlich zunahm (und gleichzeitig die Ost-West-Unterschiede deutlich abnahmen). Unverändert blieb der dominante Einfluss der Kontextmerkmale auf die Ausbildungswege, d.h. die Stadt-Land-Unterschiede im Schulweg, wohingegen der Überhang der Kontextmerkmale bei den Wegen zur Ausübung eines Ehrenamtes von 1991/92 zu 2001/02 zugunsten der Akteursmerkmale verloren ging. Dies ist u.a. auf die Erweiterung der Definition «Ehrenamt» um den Bereich der «informellen Hilfe für andere Haushalte» zurückzuführen, so dass die zuvor bestehenden OstWest-Unterschiede zurückgingen.

Während Wegezeiten für private Kontakte, Gespräche und Geselligkeit und ganz besonders Wegezeiten für Freizeitaktivitäten 1991/92 dadurch gekennzeichnet waren, dass sie in den neuen Ländern - wenn sie überhaupt stattfanden - und in den Verdichtungsregionen deutlich mehr Zeit beanspruchten als in den alten Ländern und in Gebieten außerhalb der Verdichtungsregionen, so hatte sich 2001/02 die Situation umgekehrt. Es wurden zwar in den neuen Ländern längere Wege für Freizeitzwecke zurückgelegt als im Westen, jedoch sind mittlerweile die Unterschiede zwischen den Haushaltsformen noch größer als zwischen den Regionen.

Grundsätzlich ist zu erkennen, dass für Wegezeiten zu Bildungsstätten, aber auch zu privaten Zielen Eigenschaften des Kontexts, d.h. Lage, Verkehrsanbindung und Ausstattungsgrad der Wohnorte von wesentlicher Bedeutung sind. Der Zeitaufwand für Erwerbstätigkeit, Haushalt und Kinderbetreuung unterliegt dagegen eher Differenzen durch Merkmale der Akteure. Diese lassen sich zu großen Teilen auf die geschlechtsrollentypische Verteilung der alltäglichen Aufgaben zwischen männlicher Produktions- und weiblicher Reproduktionsarbeit zurückführen, die vor allem in den alten Ländern noch häufig anzutreffen ist. Insgesamt unterliegt der Einfluss der Kontextmerkmale auf Wegezeiten über die betrachteten zehn Jahre einem Rückgang, was daran liegt, dass sich bei zahlreichen Wegezeiten die Verhältnisse im Osten denen des Westens angeglichen haben. Hintergrund dieser Entwicklung sind im Wesentlichen zwei ineinander greifende Prozesse: die Suburbanisierung in den neuen Ländern sowie die Motorisierung der Haushalte im Osten (vor allem der erwerbstätigen Personen) bei einem gleichzeitigen Rückgang der Nutzung des ÖPNVs (vgl. auch DEITERS 2000, 2001; LÖTSCher, MAYER \& MONheIm 2001), der zum einen auf die ehemals kompaktere Siedlungsstruktur ausgerichtet war und zum anderen als geradezu stigmatisierte («staatlich verordnete») Mobilitätsform nicht mit dem Automobil, dem Symbol der individuellen Freiheit, konkurrieren konnte (vgl. auch DeITERS 2000).

Für eine Zusammenschau der hier gewonnenen empirischen Ergebnisse wurden in Abb. 6 sowohl die regionalen Disparitäten der Verkehrsmittelnutzung, die eng mit den regionalen Disparitäten der Wegezeiten verbunden sind, als auch die Unterschiede in Wegezeiten und Wegstrecken dargestellt. In diesen schematisierten Darstellungen wurden die zahlreichen Detailanalysen zusammengetragen und auf die wesentlichen Muster reduziert. Während in den Kernstädten im Verdichtungsraum noch relativ hohe Anteile der alltäglichen Wege mit dem ÖPNV zurückgelegt werden, steigt mit abnehmendem zentralörtlichem Rang sowohl die Nutzung des PKWs (vgl. Motzkus 2001) als auch die des unmotorisierten Verkehrs. Die Anteile der unmotorisierten Wege nehmen allerdings in den sehr kleinen peripheren Gemeinden wieder etwas ab. Betrachtet man nun das Wechselspiel zwischen räumlichen Distanzen (Wegstrecken) und Wegezeiten unter Berücksichtigung häufig eingesetzter Verkehrsmittel, so zeigt sich, dass entscheidend ist, wie sich räumlich-metrische Wegstrecken durch die vorrangige Nutzung bestimmter Verkehrsmittel verändern. Kürzere Wegstrecken erhöhen sich dort zu langen Wegezeiten, wo häufig der ÖPNV genutzt wird, d.h. in den Kernstädten und verringern sich dort zu kurzen Wegen, wo häufig der - relativ ungehinderte - PKW zum Einsatz kommt. Ist zudem eine Vielzahl von Infrastruktureinrichtungen unmotorisiert erreichbar, wie dies z.B. in den kleineren Mittelzentren der ländlichen Regionen oder der Regionen mit Verdichtungsansätzen der Fall ist, dann erreichen dort die Wegezeiten der Befragten in der Summe die niedrigsten Werte. Diese Ergebnisse bestätigen sich im Wesentlichen auch für die unterschiedlichen Akteurstypen (vollzeiterwerbstätige Singles, Rentner und Rentnerinnen usw.). Es gibt sie also, die Orte der (zeitlich) kurzen Wege: die kleineren Zentren außerhalb der Verdichtungsregionen und die Orte der (zeitlich) langen Wege: die Kernstädte selbst.

In der Literatur standen bisher entweder die metrischen Wegelängen, der Verkehrsaufwand (z.B. in Personenkilometern) oder die Fahrleistungen (mit einzelnen Verkehrsmitteln) im Vordergrund (HoLz-RAU 1997; Kagermeier 1997; Lötscher, Mayer \& MoNheim 2001; Motzkus 2001). Holz-Rau (1997) kam dabei zu dem Schluss, dass in den kleinen Gemeinden 

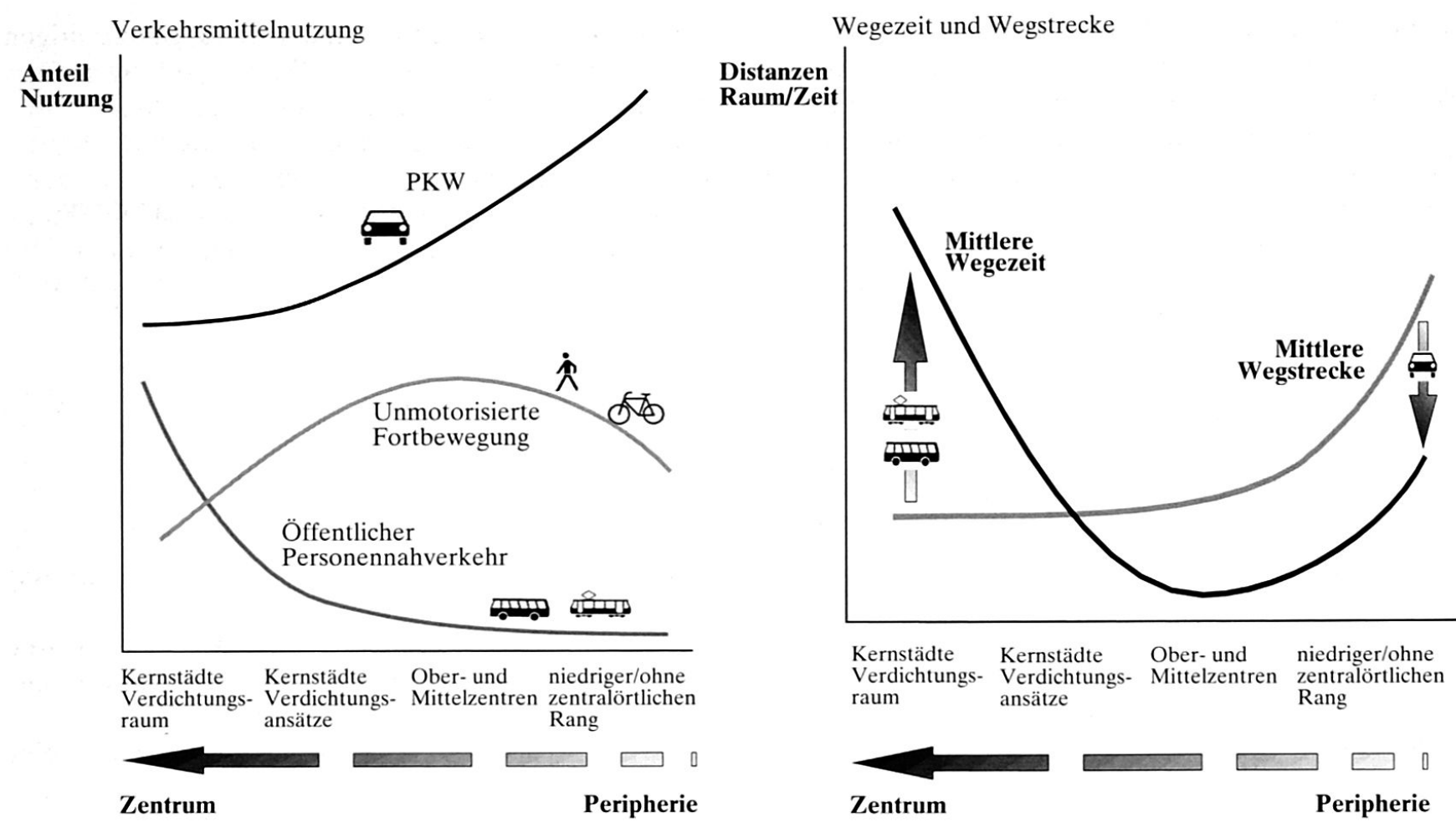

Abb. 6: Regionale Disparitäten in der Verkehrsmittelnutzung sowie in den Wegezeiten und Wegstrecken in Abhängigkeit von den genutzten Verkehrsmitteln (schematische Darstellung)

Regional disparity of transport use, trip duration and path distance according to selected transport means (schematic presentation)

Disparités régionales dans le recours aux moyens de transport, la durée et la longueur du trajet, en rapport avec les moyens de transport utilisés (représentation schématique)

Graphik: C. KRAMER

im Umfeld der Großstädte relativ große Distanzen zurückgelegt werden und dies Ergebnis einer Ursache-Wirkungs-Beziehung zwischen dem Wohnstandort und der Mobilität darstelle. Auch Kagermeier (1997) stellte fest, dass die Verkehrsleistung sowie die Anteile des motorisierten Individualverkehrs mit der Größe des Wohnorts abnahmen. In seinen Untersuchungen schnitten hinsichtlich der Kilometerleistung je Haushaltsmitglied mittelzentrale Orte sowohl innerhalb als auch außerhalb des Verdichtungsraum am günstigsten $a b$. Dieses letztgenannte Ergebnis lässt sich bei einer Fokussierung auf die Wegezeit als zentralen Indikator bestätigen, dagegen sind Orte der zeitlich langen Wege eher Orte der räumlich kurzen Wege (nämlich zentrale Orte) und umgekehrt Orte der zeitlich kurzen Wege eher Orte der räumlich langen Wege (periphere Orte bzw. kleine Zentren außerhalb der Verdichtungsregionen), in denen die meisten Wege mit dem PKW zurückgelegt werden. Die Verwendung der Wegezeiten als zentralen Indikator verändert die Perspektive insofern, dass dadurch die Effekte der vorwiegend zum Einsatz kommenden Verkehrsmittel in die Betrachtung miteinbezogen werden.

\section{Ausblick}

Die hier vorgestellte Analyse des Ausschnitts Zeitverwendung für Mobilität aus dem alltäglichen Zeitbudget der Befragten zeigt, dass regionale Unterschiede hinsichtlich der Zeitverwendung bestehen, und sie im Wechselspiel mit den Eigenschaften der Akteure zu betrachten sind. Im Detail sind dabei sich wechselseitig verstärkende Effekte, aber auch kompensatorische Effekte festzustellen. Die Verwendung des Zeitaufwandes als stellvertretender Indikator für räumliche Distanz und der Einsatz von räumlichen Typisierungen (anstelle konkreter Verortungen) bei der Nutzung der Zeitbudgeterhebungen ermöglicht erstmals Analysen auf bundesweiter Ebene. Die theoretische Erweiterung der klassischen time geography und der constraints um die Perspektive des action setting und die damit beidseitig rückgekoppelte Bedingtheit von Kontext (von Akteuren gestaltet und im probabilistischen Sinne verstanden) und Akteuren erlaubt einen neuen Zugang zu empirischen Analysen der Zeitverwendung. Der hier vorgestellte Zeitvergleich der Mobilitätszeit der Deutschen und ihrer Entwicklung 
zwischen 1991/92 und 2001/02 wurde in dem gesamten Projekt um eine ausführliche Analyse der subjektiven Wahrnehmungen und Bewertungen der Wegezeit (mental travelling times) erweitert (KRAMER 2004). Dabei zeigte sich, dass es neben den (zeit-)rationalen Argumenten, die durch die Analyse der quantitativen Daten beleuchtet werden konnten, auch eine Reihe qualitativer Aspekte gibt, die in die Analyse und Interpretation der Mobilitätszeiten einbezogen werden müssen.

Ohne die zeitlichen Ausprägungen der raumrelevanten Aktivitäten der Handelnden bleiben die Analysen ihrer Alltagswelt und deren Mobilität unvollständig. Datengrundlagen, wie die bundesdeutschen Zeitbudgetstudien bieten eine Möglichkeit, dieses timespace lag aufzuholen.

\section{Literatur}

BARKer, R.G. (1968): Ecological Psychology: Concepts and Methods for Studying the Environment of Human Behavior. - Stanford: University Press.

BLASS, W. (1980): Zeitbudget-Forschung. - Frankfurt am Main, New York: Campus.

Blotevogel, H.H. (1995): Raum. - In: Akademie FÜR RAUMFORSCHUNG UND LANDESPLANUNG (Hrsg.): Handwörterbuch der Raumordnung. - Hannover: Jänecke: 733-740.

BUNDESAMT Für BAUWESEN UND RAUMORDNUNG (Hrsg.) (1998): Indikatoren und Karten zur Raumentwicklung. Version 1.0.31 (Daten). - Bonn: Bundesamt für Bauwesen und Raumordnung.

BUNDESMINISTERIUM Für VerKeHR, BAU- UND WOHNUNGSWESEN (Hrsg.) (1976, 1982, 1989, 2002): Kontinuierliche Erhebung zum Verkehrsverhalten (KONTIV). - Berlin, http://www.kontiv2002.de. 19.3.2004.

BUNDESMINISTERIUM FÜR VERKEHR, BAU- UND WOHNUNGSWESEN (Hrsg.) (2002): Mobilitätspanel (MOP). - Berlin, http://mobilitaetspanel.ifv.uni-karlsruhe.de. 19.3.2004.

Carlstein, T., Parkes, D. \& N. Thrift (Hrsg.) (1978): Timing Space and Spacing Time (Vol. 1-3). Making Sense of Time (Vol. 1), Human Activity and Time Geography (Vol. 2), Time and Regional Dynamics (Vol. 3). - London: Arnold.

Carlstein, T. (1981): The Sociology of Structuration in Time and Space: A Time-Geographic Assessment of Giddens's Theory. - In: Svensk Geografisk Årsbok 57: 41-57.

DeITERS, J. (2000): Traffic infrastructure, car mobility and public transport. - In: MAYR, A. \& W. TAUBMANN (Hrsg.): Germany Ten Years after Reunification. Beiträge zur Regionalen Geographie 52, Leipzig: 117-137.

Deiters, J. (2001): ÖPNV in Städten und Stadtregionen. - In: INSTITUT FÜr LÄNDERKUNDE (Hrsg.): Natio- nalatlas Bundesrepublik Deutschland, Bd. 9, Verkehr und Kommunikation. - Heidelberg u.a.: Spektrum Akademischer Verlag: 68-71.

Deiters, J., GräF, P. \& G. LÖFfler (2001): Verkehr und Kommunikation - Eine Einführung. - In: INSTITUT FÜr LÄNDERKUNDE (Hrsg.): Nationalatlas Bundesrepublik Deutschland, Bd. 9, Verkehr und Kommunikation, Heidelberg u.a.: Spektrum Akademischer Verlag: 12-29.

EberLing, M. (1999): Soziale und ökologische Folgen der Arbeitszeitflexibilisierung. - Vortragsreihe «Die Zeichen der Zeit», http://www.hgdoe.de/pol/eber.htm. 10.12.2003.

EberLing, M. (2002): Wer macht die Zeit? Gesellschaftliche Zeitstrukturen im Wandel. - In: HENCKEL, D. \& M. EberLING (2002): Raumzeitpolitik. - Opladen: 189-208.

Garhammer, M. (1996): Balanceakt Zeit. Auswirkungen flexibler Arbeitszeiten auf Alltag, Freizeit und Familie. - Berlin: Sigma.

GidDENS, A. (1995): Strukturation und sozialer Wandel. - In: Müller, H.-P. \& M. Schmid (Hrsg.): Sozialer Wandel. - Frankfurt am Main: 151-191.

Guggenberger, B. (1997): Das digitale Nirwana. Hamburg: Rowohlt.

Hägerstrand, T. (1970): What about People in Regional Science. - In: Papers of the Regional Science Association 24: 7-21.

Hägerstrand, T. (1975): Space, Time and Human Conditions. - In: Karlevist, A., Lundevist, L. \& F. SNICKARS (Hrsg.): Dynamic Allocation of Urban Space. - Farnborough: 3-12.

Hägerstrand, T. (1978): Survival and Arena. - In: Carlstein, T., Parkes, D. \& N. Thrift (Hrsg.): Timing Space and Spacing Time (Vol. 2). - London: 121-145.

Hallin, O. (1991): New Paths for Time-Geography? In: Geografiska Annaler 73B, 3: 199-207.

Henckel, D. et al. (1989): Zeitstrukturen und Stadtentwicklung. $-=$ Schriften des Deutschen Instituts für Urbanistik 81, Stuttgart, Berlin, Köln, Mainz.

HENCKEL, D. et al. (Hrsg.) (1997): Entscheidungsfelder städtischer Zukunft. - = Schriften des Deutschen Instituts für Urbanistik 90, Stuttgart, Berlin, Köln.

Henckel, D. (2001): Wer verteilt die Zeit? Oder: Zwei Hände voll Wind. - In: Henckel, D. \& M. EberLing (2002): Raumzeitpolitik. - Opladen: 209-230.

Henckel, D. \& M. Eberling (2002) (Hrsg.): Raumzeitpolitik. - Opladen: Leske \& Budrich.

Holz-RAU, C. (1997): Siedlungsstrukturen und Verkehr. $-=$ Schriftenreihe der Bundesforschungsanstalt für Landeskunde und Raumordnung 84, Bonn.

INSTITUT FÜR LäNDERKUNDE (Hrsg.): Nationalatlas Bundesrepublik Deutschland, Bd. 9, Verkehr und Kommunikation. - Heidelberg u.a.: Spektrum Akademischer Verlag.

JANELle, D.G. (1995): Metropolitan Expansion, Telecommuting, and Transportation. - In: Hanson, S. 
(Hrsg.): The Geography of Urban Transportation. New York, London: 407-434.

Kagermeier, A. (1997): Siedlungsstruktur und Verkehrsmobilität. - Dortmunder Vertrieb für Bau- und Planungsliteratur, Verkehr spezial 3, Dortmund.

KLINGBeIL, D. (1978): Aktionsräume im Verdichtungsraum. Zeitpotentiale und ihre räumliche Nutzung. - = Münchner Geographische Hefte 41, München.

Klingbeil, D. (1980): Zeit als Prozess und Ressource in der sozialwissenschaftlichen Humangeographie. In: Geographische Zeitschrift 68, 1: 1-32.

Kramer, C. (2000): Zeit und Raum - Zeit für Raum? Räumliche Disparitäten in der individuellen Zeitverwendung - ein Forschungsprojekt. - In: EHLING, M. \& J. Merz (Hrsg.): Zeitbudget in Deutschland - Erfahrungsberichte der Wissenschaft. - = Schriftenreihe Spektrum der Bundesstatistik 17, Wiesbaden: 19-43.

Kramer, C. (2004):Verkehrsverhalten und Mobilität. In: Statistisches Bundesamt (Hrsg.): Alltag in Deutschland -Analysen zur Zeitverwendung. $-=$ Schriftenreihe Forum der Bundesstatistik 43, Stuttgart (im Druck).

Lötscher, L., Mayer, O. \& R. Monheim (2001): Entwicklung der privaten Motorisierung. - In: INSTITUT FÜR LÄNDERKUNDE (Hrsg.): Nationalatlas Bundesrepublik Deutschland, Bd. 9, Verkehr und Kommunikation. - Heidelberg u.a.: Spektrum Akademischer Verlag: 62-63.

LÖTSCHER, L., MAYER,O. \& R. MONHEIM (2001): Mobilität und Verkehrsmittel. - In: INSTITUT Für LÄNDERKUNDE (Hrsg.): Nationalatlas Bundesrepublik Deutschland, Bd. 9, Verkehr und Kommunikation. - Heidelberg u.a.: Spektrum Akademischer Verlag: 58-61.

LüDTKE, H. (1995): Zeitverwendung und Lebensstile. - = Marburger Beiträge zur Sozialwissenschaftlichen Forschung 5, Marburg.

MotzkuS, A. (2001):Räumliche Struktur des Pkw-Verkehrs. - In: INSTITUT FÜR LäNDERKUNDE (Hrsg.): Nationalatlas Bundesrepublik Deutschland, Bd. 9, Verkehr und Kommunikation. - Heidelberg u.a.: Spektrum Akademischer Verlag: 64-65.

MüCKENBERGER, U. (1999): Kommunale Zeitplanung in Deutschland - die Beispiele Hamburg, Bremen und Hannover. - Vortragsreihe "Die Zeichen der Zeit", http://www.hgdoe.de/pol/muecke.htm. 18.3.2004.

Mückenberger, U. (2001): Örtliche Zeitkonflikte und die Macht der Zivilgesellschaft. - In: Henckel, D. \& M. Eberling (2002): Raumzeitpolitik. - Opladen: 231-250

MülleR-WiChmanN, C. (1984): Zeitnot. Untersuchungen zum «Freizeitproblem» und seiner pädagogischen Zugänglichkeit. - Weinheim, Basel: Beltz.

RAMmSTEDT, O. (1975): Alltagsbewußtsein von Zeit. In: Kölner Zeitschrift für Soziologie und Sozialpsychologie 27: 47-63.

RINDERSPACHER, J.P. (1985): Gesellschaft ohne Zeit. Individuelle Zeitverwendung und soziale Organisation der Arbeit. - Schriften des Wissenschaftszen- trums Berlin, Internationales Institut für Vergleichende Gesellschaftsforschung/Arbeitspolitik, Frankfurt am Main, New York: Campus.

Schallaböск, K.O. (1991): Verkehrsvermeidungspotentiale durch Reduktion von Wegezahlen und Entfernungen. - In: Informationen zur Raumentwicklung 1/2: 67-84.

SchallaböcK, K.O. (1998): Zukunftschance Mobilität. - In: «mobilität für das 21.jahrhundert!». - Dokumentation der Verkehrskonferenz der Grünen Vorarlbergs, Feldkirch, 13.-15.9.1998, http://vorarlberg.gruene.at/ publikationen/mobilitaet/schallaboeck.htm. 4.4.2004.

SchмiтZ, S. (2001): Revolutionen der Erreichbarkeit. - = Stadtforschung aktuell 83, Opladen: Leske + Budrich.

SCHREINER, J. (1995): Aktionsraumforschung auf phänomenologischer und handlungstheoretischer Grundlage. - Geographische Zeitschrift 86, 1: 50-66.

SHEN, Q. (2000): Spatial and Social Dimensions of Commuting. - In: Journal of the American Planning Association 66, 1: 68-84.

Statistisches Bundesamt (Hrsg.) (1998, 2003): Bundesdeutsche Zeitbudgeterhebung (Datensatz). - Wiesbaden.

Turner, J. \& M. Grieco (1998): Gender and time poverty: the neglected social policy implications of gendered time, transport and travel. - In: Time and Society 9, 1: 129-137.

Viriolo, P. (1992): Rasender Stillstand. - München, Wien: Hanser.

WeichHart, P. (2003): Gesellschaftlicher Metabolismus und Action Settings. Die Verknüpfung von Sach- und Sozialstrukturen im alltagsweltlichen Handeln. - In: Meusburger, P. \& T. SchWAN (Hrsg.): Humanökologie. - = Erdkundliches Wissen 135, Wiesbaden: 15-44.

Werlen, B. (2000): Sozialgeographie. - Bern, Stuttgart, Wien: UTB.

Wolf, K. \& C.M. Scholz (1999): Neue Zeitverwendungsstrukturen und ihre Konsequenzen für die Raumordnung. - = Forschungs- und Sitzungsberichte der Akademie für Raumforschung und Landesplanung 207, Hannover.

ZiERHOFER, W. (1989): Alltagsroutinen von Erwachsenen und Erfahrungsmöglichkeiten von Schulkindern. - In: Geographica Helvetica 44, 2: 87-92.

\section{Zusammenfassung: Deutschland unterwegs - Zeitver- wendung für Mobilität im wiedervereinten Deutsch- land}

Der vorliegende Beitrag beschäftigt sich mit der Mobilitätszeit im wiedervereinten Deutschland im Sinne einer theoretisch erweiterten time geography. Insbesondere ist von Interesse, an welchen Orten Menschen viel oder wenig Zeit für ihre alltägliche Mobilität einsetzen (müssen). Eine Grundhypothese der Arbeit ist, dass Zeit als Ressource nicht nur sozial, sondern 
auch räumlich ungleich verteilt ist. Es stellt sich somit die Frage, inwieweit sich Mobilitätszeiten unter dem Einfluss des (z.T. auch konstruierten) Kontexts, im Sinne des räumlichen Umfelds eines Individuums, zusammen mit akteursspezifischen Merkmalen (Lebenssituation, Familie) auf die Mobilitätszeiten und die genutzten Verkehrsmittel verändern. Im Zentrum der empirischen Arbeiten steht die Auswertung der beiden bundesdeutschen Zeitbudgetstudien des Statistischen Bundesamtes von 1991/92 und 2001/02. Dabei zeigt sich u.a., dass in dem beobachteten Jahrzehnt insgesamt sowohl der Zeitaufwand für Mobilität als auch der Anteil der Personen, die Wege ausüben, deutlich zunehmen und (noch) kein Rückgang der realen Mobilität zugunsten einer virtuellen Mobilität stattfindet.

\section{Summary: Germany on the Go! Time invested in mobility in a unified Germany}

This paper deals with the time invested in mobility in unified Germany from the point of view of a theoretically expanded time geography. Of particular interest is the definition of places where people (must) invest more or less time during their daily mobility. Underlying the investigation is the basic hypothesis that the availability of time is both socially and spatially unequally distributed. Thus, a particular focus is the influence of the (in part constructed) context (i.e. the spatial surroundings of an individual) and actor specific characteristics (for example current situation or family) on changes in mobility duration and selection of transport means. Central to the investigation was the evaluation of two federal time budget surveys carried out by the Federal Bureau of Statistics in 1991/1992 and 2001/2002. Particularly noticeable between the two surveys is the strong increase in time invested in mobility and in the number of mobile persons. Further, there appears as yet to be no decrease in actual mobility in favour of virtual mobility.

\section{Résumé: L'Allemagne en déplacement - Durée de la mobilité dans l'Allemagne réunifiée}

La présente contribution porte sur la durée de la mobilité dans l'Allemagne réunifiée, en tant que base d'une réflexion théorique élargie, relative à la géographie du temps. Il est notamment intéressant de connaître les lieux où les hommes doivent consacrer plus ou moins de temps à leur mobilité quotidienne. Une hypothèse fondamentale du travail réside dans le fait que le temps considéré comme ressource n'est pas également réparti dans l'espace et dans la société. La question est ainsi posée de savoir dans quelle mesure les temps de mobilité sont influencés par le contexte, construit ou non, dans le sens de l'environnement spatial d'un individu, en liaison avec des caractéristiques spécifiques aux acteurs (niveau de vie, situation familiale), et dans quelle mesure ils modifient aussi les moyens de transport utilisés. Au centre des travaux empiriques se situe l'élaboration des résultats inhérents aux deux études fédérales allemandes consacrées par l'Office fédéral de statistique au budget-temps, en 1991/92 et 2001/02. Ces données révèlent que durant la décennie observée, tant en ce qui concerne le temps consacré à la mobilité que pour ce qui est de la part des personnes en déplacement, le temps de mobilité augmente sensiblement. Aucune régression de la mobilité actuelle n'est (encore) à enregistrer au profit de la mobilité virtuelle.

\section{Didaktische Hinweise}

- Welche theoretischen Konzepte gibt es bisher, um Zeit und Raum geographisch zu betrachten?

- Welche Unterschiede zeigen sich hinsichtlich Wegezeit und Anteil der Ausübenden nach Stadt-LandDifferenzen und zwischen den neuen und alten Ländern und wie lässt sich dies erklären?

- Welche Zusammenhänge bestehen zwischen der Verkehrsmittelnutzung und den mittleren Wegezeiten und Wegstrecken bei einer Betrachtung, die nach Zentrum und Peripherie differenziert?

PD Dr. Caroline Kramer, Ludwig-Maximilians-Universität, Department für Geo- und Umweltwissenschaften, Sektion Geographie, Luisenstr. 37, D-80333 München.

e-mail: caroline.kramer@ssg.geo.uni-muenchen.de

\section{Manuskripteingang/received/manuscrit entré le 5.1.2004}

Annahme zum Druck/accepted for publication/accepté pour l'impression: 24.5 .2004 